\title{
Congenital Heart Disease and Maternal Diabetes Mellitus
}

\author{
A. C. Petropoulos ${ }^{* 1,2}$, A. Xudiyeva ${ }^{1}$ and M. Ismaylova ${ }^{1}$ \\ ${ }^{1}$ Merkezi Klinika, 76 Parlament Prospekti, Bakı AZ1006, Azerbaijan \\ ${ }^{2}$ Azerbaijan Medical University, Samad Vurğun Baku, Azerbaijan
}

\begin{abstract}
Introduction: Diabetes mellitus is a relatively common illness that can complicatepregnancy and result in an increased incidence of congenital malformations.Offspring of diabetic mothers suffering from type IDDM have a fivefold incidence of congenital malformations compared to pregnancies in the general healthy population. Specifically, the pattern of congenital heart disease(CHD) encountered among this group, with an emphasis on abnormalities of laterality, looping and conotruncal septation, suggesting that the maternal metabolic state affects cardiogenesis at a very early stage of the developmental period, prior to 7 weeks of gestation. Although many have been written on the effect of diabetes in pregnant women, less is known for the effects of type II DM and gestational diabetes mellitus(GDM) and its role in provoking $\mathrm{CHD}$.
\end{abstract}

Aim: Aim of this paper is to review the literature regarding the types of CHD seen in offspring of mothers suffering fromdifferent typesof diabetes mellitus, maternal types 1 and 2 and gestational and to comment on the incidences and any differencesfound in the types of detected CHD.

Method: a systematic literature resurge of the last 15 years was reviewed focusing to produce answers on the aims of the study.

Conclusion: basedon the existing evidencehigh frequency of CHD can be found in any type of maternal diabetes mellitus. For this reason, we believe that any type of diabetes present in a pregnancy must be a strong indication undergoing specific special fetal cardiac prenatal screening, aiming to detect possible CHD.

\section{Abbreviations}

Aov - Aortic Valve; ASD- Atrial Septal defects; AVSD - Atrioventricular Septal Defect; BMI - Body Mass Index; CoA- Coarctation of the AortaCHD - Congenital Heart Disease; DILV - Double Inlet Left Ventricle; DM - Diabetes Mellitus; DILV-Double Inlet Left Ventricle; DORVDouble Outlet Right Ventricle; ECA - Extra Cardiac Abnormality; GDM - Gestational Diabetes Mellitus; HbA1c - Glycated Hemoglobin A1c; HLHS - Hypoplastic Left Heart Syndrome; IDDM - Insulin Dependent Diabetes Mellitus; IVS - Intact Ventricular Septum; MAPCA- Multiple Aorto Pulmonary Collaterals; MV - Mitral Valve; NT -Nuchal Translucency; OGT Test - Oral Glucose Tolerance Test; PDA- Patent Ductus Arteriosus;PvAtr- Pulmonary valve Atresia; PvS- Pulmonary valve Stenosis; SV- single ventricles; SVT - Supraventricular Tachycardia;ToFTetralogy of Fallot; TAPVD - Total Anomalous Pulmonary Venous Drainage; TGA - Transposition of the Great Arteries; TA- trancus arteriosus; TvAtr- Tricuspid valve atresia; VSD - Ventricular Septal Defect.

\section{Introduction}

Many studies have proven that offspring of diabetic mothers have a fivefold incidence of congenital malformations compared to pregnancies in the general healthy population [1,2].In the mid 1980's the United Kingdom, the Diabetes Pregnancy Survey reported the presence of major congenital malformations, of which congenital heart disease(CHD) constituted a significant element of them. Further CHD are the most important single causes of perinatal mortality amongst the offspring of diabetic mothers $[3,4]$. Other congenital malformations linked to maternal diabetes include Central Nervous System malformations that have been found 16 times more frequently seen in maternal Insulin Dependent Diabetes Mellitus(IDDM) or type I diabetes mellitus. In particular, the risk of anencephaly is 13 times higher, whereas the risk of spina bifida is 20 times higher. The risk of caudal dysplasia is up to 600 times higher in these infants [5]. Others, consist of Renal: hydronephrosis, renal agenesis, ureteral duplication, Ear abnormalities, Gastrointestinal: duodenal or anorectal atresia and small left colon syndrome [6]. Further to the aforementioned, other conditions as respiratory distress syndromegrowth abnormalities such as macrosomia (large for gestational age) and microsomia (small for gestational age), hyperviscosity syndrome secondary to polycythemia,hypoglycemic episodes due to unstable glucose compensating regulation, hypocalcemia, hypomagnesemia, and iron deficiency anemia increase morbidity and mortality risks in the immediate postnatal period [6].

\section{Publication History:}

Received: December 21, 2015 Accepted: June 15, 2016

Published: June 17, 2016

\section{Keywords:}

Maternal diabetes mellitus, Gestational diabetes, Fetal cardiac screening, Congenital heart diseases

In 2014 World Health Organization reported a 9\% worldwide prevalence of diabetes mellitus(DM)in people over 18 years old. From this cohort $90 \%$ suffer from type II diabetes mellitus [7]. Recent data show that gestational diabetes mellitus (GDM) prevalence has increased by approximately $10-100 \%$ in several race/ethnicity groups during the past 20 years. Scientific awareness about the overlapping of cases of undiagnosed diabetes mellitus type 2 and GDM has been raised in the past [8]. By definition GDM develops in the second or third trimester of pregnancy, in pregnancies that are screened and found to have a normal level of fasting glucose and have none carbohydrate intolerance or have not been screened but thought to have no evidence of carbohydrate intolerance in the first trimester $[8,9]$. GDM, by many studies has been attributed to an increase in perinatal morbidity and mortality [9].

It has been estimated that abnormal glucose regulation and control rangein $3-10 \%$ of all pregnancies. While $80-88 \%$ out of these cases

"Corresponding Author: Prof. Andreas Petropoulos, Azerbaijan Medical University, Baku, Azerbaijan, E-mail: andrepetropoulos@gmail.com

Citation: Petropoulos AC, Xudiyeva A, Ismaylova M (2016) Congenital Heart Disease and Maternal Diabetes Mellitus. Int J Diabetes Clin Diagn 3: 118. doi: http://dx.doi.org/10.15344/2394-1499/2016/118

Copyright: @ 2016 Petropoulos et al. This is an open-access article distributed under the terms of the Creative Commons Attribution License, which permits unrestricted use, distribution, and reproduction in any medium, provided the original author and source are credited. 
Citation: Katagiri N, Kigawa I, Yahara Y, Ueda Y, Kaneko S, et al. (2016) Liraglutide is a Perioperative Therapeutic Option for Patients with Type 2 Diabetes that Undergo Elective Surgery. Int J Diabetes Clin Diagn 3: 117. doi: http://dx.doi.org/10.15344/2394-1499/2016/117

Page 2 of 5

are related to abnormal glucose control of pregnancy known as GDM. This type of diabetes seems to be the far more common form among pregnant, leaving IDDM representing 4-6\% and type 2, 8-12 $\%$ of pregnancies of women affected from DM. Furthermore, women known to have a normal glucose tolerance and fasting levels of glucose and present with GDM, demonstrate an increased risk of type $2 \mathrm{DM}$ in the future [10].

\section{Method}

A systematic literature review on papers published regarding the incidence, anatomical type and mechanism that produces CHD in different types of DM was used to address the aims of this paper.

\section{Congenital heart disease seen among pregnant with different types} of diabetes mellitus

The correlation of CHD and diabetes mellitus is well documented in many series since the early period of fetal echocardiography back in the early 1970's[11]. Although the most common CHD seen in mostly all studies that address cardiac defects in offspring of mothers suffering from any form of DM is hypertrophic cardiomyopathy secondary to the maternal diabetes[12] a large range of other CHD are also reported.

Although diabetic cardiomyopathy is not recognized as a structural malformation due to its often-limiting clinical consequence to the heart, it is far the most common presentation from the cardiovascular system.An incidence rate of $30 \%$ in offspring of mothers suffering from IDDM has been reported. In prevalence in pregnancies complicated from type $2 \mathrm{DM}$ and GDM was less recognized[13]. It is characterized by thickening of the interventricular septum and ventricular walls, and by systolic and diastolic dysfunction of the neonatal heart. This condition is normally asymptomatic in utero and may only result in congestive heart failure in the immediate postnatal period, although this is uncommon and transient[14].

Many studies underline an increase incidence of CHD as high as is $3-6 \%$ in offspring's of diabetic mothers. It is five times higher than in normal pregnancies and commonly includes complex lesions such as conotruncal abnormalities as transposition of the great arteries (TGA), tricuspid atresia (TvAtr), and truncus arteriosus(TA) have been reported more frequently [15]. Specifically, the frequency of TGA in live born babies of mothers with pre-existing diabetes is 17 times more than that in normal population [16]. The closure of ductus arteriosus and postnatal decrease in pulmonary artery pressure are delayed in IDMs when compared with control infants during the first days of life [17]. This increase incidence of patent ductus arteriosus (PDA) has also been shown in cohorts of neonates of mothers suffering from IDDM [18]. Additional to the more common forms discredited previously, two large and recent series by Lisowski et al. and Hunter et al. describe a larger spectrum of CHD. These, in an incident categorization include ventricular septal defects(VSD), single verticals(SV),total atrioventricular septal defects(AVSD), atrial septal defects, Hypoplastic Left Heart Syndrome (HLHS), Coarctation of the Aorta(CoA), Pulmonary and Aortic valve stenosis (PvS),(AovS).Heterotaxia syndromes are reported in both series. Finally, in both series although presented, the following defects had a minor percentage: total and partial anomalous pulmonary vein drainage(TAPVD), Shon's syndrome and double inlet left ventricle(DILV) with PvAtr and TAPVD $[15,19]$.

\section{Discussion}

Although perinatal mortality has declined dramatically in the developed and developing countriesduring the latest decades, in diabetic pregnancies, most studies of large populations continue to encounter a higher mortality among these patients than in control populations. Additionally,poorly controlled diabetes mellitus, is known to be associated with congenital abnormalities, including CHD [15,16,19,20,21].

From studies throughout the 1990's until resent times the prevalence of both hypertrophic cardiomyopathy and CHD related to pregnancies of IDDM mothers been reported from 6.0 to $8.5 \%[15,22]$. This high incidence seems to fall in the latest study by Hunter to 3.6 to $3.1 \%$ [19]. This is probable due to the higher alertness andscreening detection in the early stages of pregnancies complicated with IDDM or DM type II.

Regarding the types of CHD seen related with IDDM, DM type II and GDM a consensus among the researches exists. Most frequently complex CHD seen areconotruncal defects as TGA, ToF and TA,TvAtr, visceral heterotaxia syndromes, SV, and DORV or DILV with PvAtr. Also extremely frequent was the presence of Hypertrophic Cardiomyopathy liked to DM [15,19,21]. Remarkable nearby all studies show that common defects like PDA, ASD,AVSD, PvS,CoA,AovS, and when arrhythmias were involved Supra Ventricular Arrhythmia(SVT) are common [15,16,18,19,22]. This observation minimizes the ability to correlate these simple CHD to undetected DM of any type as they are similar present in the pool of CHD in pregnancies not effected by DM $[11,15,19]$.

The exact mechanism that produces CHD in the settings of any type of DM, seems to multifactorial and not still fully defined. Animal models have demonstrated that diabetic embryopathy is a complex process influenced by metabolic signaling, cell signaling, maternal and fetal genotypes and environmental factors as well as exposure touncontrolledhyperglycemia. This last metabolic condition shows to influence changes in the fetal metabolic and circulatory homeostasis. This results in a more hypoxemic environment, that can provoke in very early stages of the pregnancy sever forms of CHD. In non-diabetic pregnancies there is an increase in maternal insulin resistance due to maternal physiological adaptations which occur to ensure adequate fetal growth and development [23]. These adaptations include maternal glucose intolerance, altered glucose metabolism, cortisol/ growth hormone levels and may be compounded by reduced physical activity and increased calorific intake during pregnancy.GDM, which tends to develop in the 2nd or 3rd trimester of pregnancy, has been attributed to an increase in perinatal morbidity and mortality, although pregestational diabetes is known to have a greater association with fetal anomalies than GDM $[19,21,23]$. The occurrence of CHD in pregnancies complicated by GDM may reflect a combination of hyperglycemia, insulin resistance, an elevated BMI and possibly most significantly, undiagnosed pre-gestational diabetes [19].

In the most resent published study by Hunter et.al.the risk of CHD in a GDM pregnancy was $2.76 \%$ with an additional risk of $26.7 \%$ for a concomitant extra cardiac abnormality. This was similar to the incidence of CHD in IDDM pregnancies, with $3.1 \%$ to present any type of CHD, and a risk of concomitant extra cardiac abnormalities in $25 \%$ of cases [19]. Not only the incidence of $3.6 \%$ but also the anatomical types of the different CHD are similar with the data published in 2010 large multi-center retrospective clinical study, 
Citation: Katagiri N, Kigawa I, Yahara Y, Ueda Y, Kaneko S, et al. (2016) Liraglutide is a Perioperative Therapeutic Option for Patients with Type 2 Diabetes that Undergo Elective Surgery. Int J Diabetes Clin Diagn 3: 117. doi: http://dx.doi.org/10.15344/2394-1499/2016/117

Page 3 of 5

literature review and meta-analysis by Lisowski [15]. Thesedata prove that all types of DM can produce a large spectrum of CHD. Although conotruncal abnormalities are more frequent nearly all types of CHD can be seen. The minor differences in the incidence between CHD seen in IDDM and DM type II or GDM can be explained by the more difficult way to control medically the IDDM hyperglycemia in relation to the other types of DM [21].

As type II DM prevalence, now days is increasing [7] and many of the cases are undetected due to poor screening pre and during pregnancy, physicians and women must be aware of the association between an increase BMI and type II DM andadverse pregnancy outcomes. As the total burden, amount of morbidity, mortality and total therapeutical finance cost of dealing with complex forms of CHD is extremely high benefits from screening at risk women for DM before they conceive. In recent years $\mathrm{WHO}$ have advised that a glycated hemoglobin Alc (HbA1c) level can be used to diagnose DM, replacing the oral glucose tolerance (OGT) test which is longer as a test and requires the patient to fast. One of the advantages of the HbAlc is it can be taken with routine bloods and a level above $6.5 \%$ confirms the diagnosis of diabetes [19]. HbA1c may allow to apply measures as weight loss, and subsequent good diabetic control to prevent CHD associated to DM. Furthermore, we have enough evidence to proclaim that pregnancies showing risk factors for type II DM or presenting with GDM carry a major indication to be offeredfetal cardiac consultation similar to those pregnancies of mothers suffering from IDDM.

\section{Conclusion}

Based on our data we reviewed from the resent existing literature, we believe that pregnancies complicated with GDM present an increased risk of producing CHD. Mechanisms of unknown or poorly controlledhyperglycemia, insulin resistance, an elevated BMI and mostly undiagnosed pre-gestational diabetes can provoke GDM. This condition can increase nearby 3 times the risk of a variety of sever complex or common CHD. Until proven otherwise, it is our belief that pregnancies complicated by type II DM and GDM must be referred for detailed fetal cardiac evaluation.

\section{Competing Interests}

None of the authors of this paper have any direct or indirect financial or personal relationships, interests and affiliations, related to the subject of the paper.

\section{References}

1. Molsted-Pedersen L, Tygstrup I, Pedersen J (1964) Congenital malformations in newborn infants of diabetic women correlation with maternal diabetic vascular complications. Lancet 1: 7343.

2. Casson IF, Clarke CA, Howard CV, McKendrick O, Pennycook S, et al (1997) Outcomes of pregnancy in insulin dependent diabetic women: results of a five year population cohort study. BMJ 315: 275-278.

3. Lowy C, Beard RW, Goldschmidt J (1986) The UK diabetic pregnancy survey. Acta Endocrinol Suppl (Copenh) 277: 86-89.

4. Becerra JE, Khoury MJ, Cordero JF, Erickson JD (1990) Diabetes mellitus during pregnancy and the risks for specific birth defects: a populationbased case-control study. Pediatrics 85: 1-9.

5. Shaw GM, Quach T, Nelson V, Carmichael SL, Schaffer DM, et al. (2003) Neural tube defects associated with maternal periconceptional dietary intake of simple sugars and glycemic index. Am J ClinNutr 78: 972-978.

6. Metzger BE, Lowe LP, Dyer AR, Trimble ER, Chaovarindr U, et al. (2008) Hyperglycemia and adverse pregnancy outcomes. N Engl J Med 358: 1991-2002.
7. Global status report on non-communicable diseases (2014) Geneva, World Health Organization, 2012.

8. Assiamira Ferrara (2007) Increasing prevalence of GDM.Diabetes Care, Vol 30, Supp. 2.

9. Balsells M, García-Patterson A, Gich I, Corcoy R (2012) Major congenital malformations in women with gestational diabetes mellitus: a systematic review and meta-analysis. Diabetes Metab Res Rev 28: 252-257.

10. Charles F Potter, Ted Rosenkrantz, (May 2013) Infant of diabetic mother; Medscape Education, Medline.

11. Allan LD, Sharland GK, Milburn A, Lockhart SM, Groves AMM, et al. (1994) Prospective Diagnosis of 1,006 Consecutive Cases of Congenital Heart Disease in the Fetus. JACC 23: 452-458.

12. Ullmo S, Vial Y, Di Bernardo S, Roth-Kleiner M, Mivelaz Y, et al. (2007) Pathologic ventricular hypertrophy in the offspring of diabetic mothers: a retrospective study. Eur Heart J 28: 1319-1325.

13. Pildes RS (1973) Infants of diabetic mothers. N Engl J Med 289: 902-904.

14. Sardesai MG, Gray AA, McGrath MM, Ford SE (2001) Fatal hypertrophic cardiomyopathyin the fetus of a woman with diabetes. ObstetGynecol 98: 925-927.

15. Lisowski LA, Verheijen PM, Copel JA, Kleinman CS, Wassink S, et al (2010) Congenital heart disease in pregnancies complicated by maternal diabetes mellitus. An international clinical collaboration, literature review, and meta-analysis. Herz 35: 19-26.

16. Wren C, Birrell G, Hawthorne G (2003) Cardiovascular malformations in infants of diabetic mothers. Heart 89: 1217-1220.

17. Vela-Huerta M, Aguilera-Lopez A, Alarcon-Santos S, Amador N, AldanaValenzuela C, et al. (2007) Cardiopulmonary adaptationin large for gestational age infants of diabetic and non-diabetic mothers. Acta Paediatr 96: 1303-1307.

18. Abu-Sulaiman RM, Subaih B (2004) Congenital Heart Disease in Infants of Diabetic Mothers: Echocardiographic Study. PediatrCardiol 25: 137-140.

19. Lindsey E Hunter, Gurleen K Sharland (2015) Maternal Gestational Diabetes and Fetal Congenital Heart Disease: An Observational Study. J Preg Child Health 2: 132

20. Avalos G, Owens L, Dunne F, for the ADIPC (2013) Applying Current ScreeningTools for Gestational Diabetes Mellitus to a European PopulationIs it Time for Change? Diabetes Care. 36: 3040-3044.

21. Hornberger LK (2006) Maternal diabetes and the fetal heart. Heart 92 1019-1021

22. RoodpeymaS, Rafieyian S, Khosravi N, Hashemi A (2013)Cardiovascula Complications in Infants of Diabetic Mothers: An Observational Study in a Pediatric Cardiology Clinic in Tehran. J Compr Ped 3: 119-23.

23. Hadden DR, McLaughlin C (2009) Normal and abnormal materna metabolism during pregnancy. Erratum in Semin Fetal Neonatal Med 14: $66-71$. 